\title{
Late presentation during the COVID-19 pandemic of severe procidentia: a high-grade poorly differentiated sarcoma of unknown origin
}

\author{
Christina Pappa 다, ${ }^{1}$ Sarah Louise Smyth 다, ${ }^{1}$ Hooman Soleymani majd (1) 1,2
}

'Department of Gynaecology Oncology, Oxford University Hospitals NHS Foundation Trust, Oxford, UK

${ }^{2}$ Nuffield Department of Women's Reproductive Health, Medical Siences Division, Oxford University, Oxford, UK

Correspondence to Dr Hooman Soleymani majd; hooman.soleymani@ouh.nhs.uk

Accepted 13 January 2022

Check for updates

(c) BMJ Publishing Group Limited 2022. No commercial re-use. See rights and permissions. Published by BMJ.

To cite: Pappa C, Smyth SL, Soleymani majd H. BMJ Case Rep 2022;15:e246710. doi:10.1136/bcr-2021246710

\section{SUMMARY}

High-grade poorly differentiated sarcomas of unknown primary origin constitute a rare entity and are characterised by wide histopathological diversity and atypical presentations. We present such an unusual case attending with severe procidentia in a 68-year-old postmenopausal female. On review of the literature, there are no similar cases reported. Herein, we present this case as in view of its rare clinical appearance combined with the advanced and histologically uncertain nature of the tumour, which raised significant challenges regarding diagnosis and surgical management in considerations of oncological hygiene and risk of tumour spillage. This was further potentiated by delay in diagnosis and treatment due to the COVID-19 pandemic.

\section{BACKGROUND}

Tumours of unknown primary origin cover a wide spectrum of neoplasms that either represent metastatic disease of an occult primary tumour or constitute a primary where it is impossible to accurately classify on histopathological examination due to the undifferentiated content. ${ }^{1}$ Malignancies of unknown primary origin comprise $3 \%-5 \%$ of all malignant neoplasms and only $5 \%$ of them represent poorly differentiated neoplasms (lymphomas, sarcomas, germ cell tumours, melanomas). ${ }^{2}$

Poorly differentiated sarcomas are rare malignant tumours deriving from the transformation of mesenchymal stem cells through a rather unclarified pathway. ${ }^{3}$ Such tumours are characterised by high heterogeneous potential, and therefore, have a potentially unpredictable prognosis with early relapse rates and high rates of metastasis. ${ }^{4}$

The undetermined background of these rare tumours has posed several limitations in the diagnostic pathways and optimal treatment. ${ }^{5}$ As a result, there are no proven effective management guidelines, with the clinical outcome depending on the personalised management approach offered by the responsible team. ${ }^{6}$ Therefore, it is of paramount importance to report on these cases, especially in consideration of such an unusual and complicated presentation.

\section{CASE PRESENTATION}

We present the case of a 68-year-old postmenopausal multiparous woman. She has a medical history of osteoarthritis and urethral caruncle and a previous surgical history of laparoscopic right hemicolectomy for Dukes stage B R0 pT3 L0 V0
N0 (0/17) moderately differentiated adenocarcinoma of the ascending colon; for which adjuvant treatment was not required. She has no known drug allergies and is a heavy smoker with a body mass index of 26.7 and a performance status of zero.

The patient presented to her general practitioner with vaginal bleeding and green discharge associated with lower abdominal discomfort. The patient stated she had experienced a 'sensation down below' for a few months and assumed this to be a prolapse. On examination, a large, incarcerated, irregular and infected, cancerous looking mass was identified; prompting a 2-week wait referral to gynaecology. On further examination, this was confirmed to be a prolapse consisting of hard, craggy and oedematous skin, which was considered as either a uterine procidentia, with a possible tumour within the uterine body, or a mass protruding through, from or surrounding the cervix. The rest of the vagina was described as unremarkable. Due to urinary retention secondary to mass effect an indwelling urinary catheter was placed.

The patient underwent CT scanning of the chest, abdomen and pelvis, which reported increased soft tissue in the region of the vagina (figure 1). The uterus was not identified and therefore presumed to have prolapsed into the vagina. The cervix was also reported to be bulky, raising the possibility of a cervical tumour acting as a cause of the prolapse. There was no evidence of lymphadenopathy or metastatic disease. A subsequent MRI scan of the pelvis reported a severe uterine prolapse making the anatomy of the uterus and cervix difficult to interpret with abnormal hyper intense tissue centred on the usual position of the cervix and restricted diffusion in keeping with a tumour. This was 9 $\mathrm{cm}$ in maximum dimension and filled the vaginal fornices circumferentially. No endometrial thickening was identified and the parametria could not be assessed. The radiological conclusion was that of a uterine prolapse with possible large exophytic cervical primary tumour despite recent completion of cervical screening (figures 2 and 3 ).

Subsequent to this, the patient underwent examination under anaesthesia. This was initially delayed due to the patient acquiring COVID-19 infection. Multiple biopsies from the mass were acquired. The friable procidentia descended $5 \mathrm{~cm}$ below the introitus and the tumour obliterated the external cervical os. Hysteroscopy was not attempted. Histology reported a high-grade malignant tumour of mesenchymal origin with extensive necrosis and 


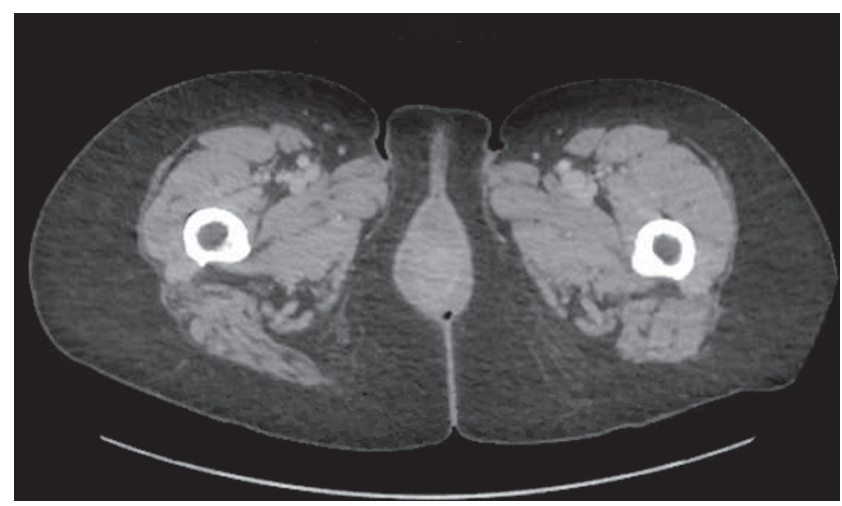

Figure 1 CT chest, abdomen and pelvis with contrast-axial view demonstrating increased soft-tissue in the region of the vagina. The uterus was not identified and was presumed prolapsed into the vagina. There was no evidence of lymphadenopathy or metastases.

high mitotic count (>50/10 high power fractions). Tumour cells contained plump ovoid and spindle-shaped nuclei. There was prominent nuclear pleomorphism and no definite gland formation, intercellular bridges or keratinisation seen. Immunohistochemistry was positive for CD56, p53, p16, vimentin, CD10, ERG (ETS-related gene (ETS - E-26 transformation-specific)) and cyclin D1 with faint positivity for CD31, CK7, SOX-10, PAX8, S100, muscle-specific actin and desmin. While considered, a neuroendocrine tumour was deemed unlikely as the CD56 staining was non-specific and there was absence of cytokeratin and synaptophysin staining. A recurrence of the previous colorectal adenocarcinoma was also ruled out, as this had been

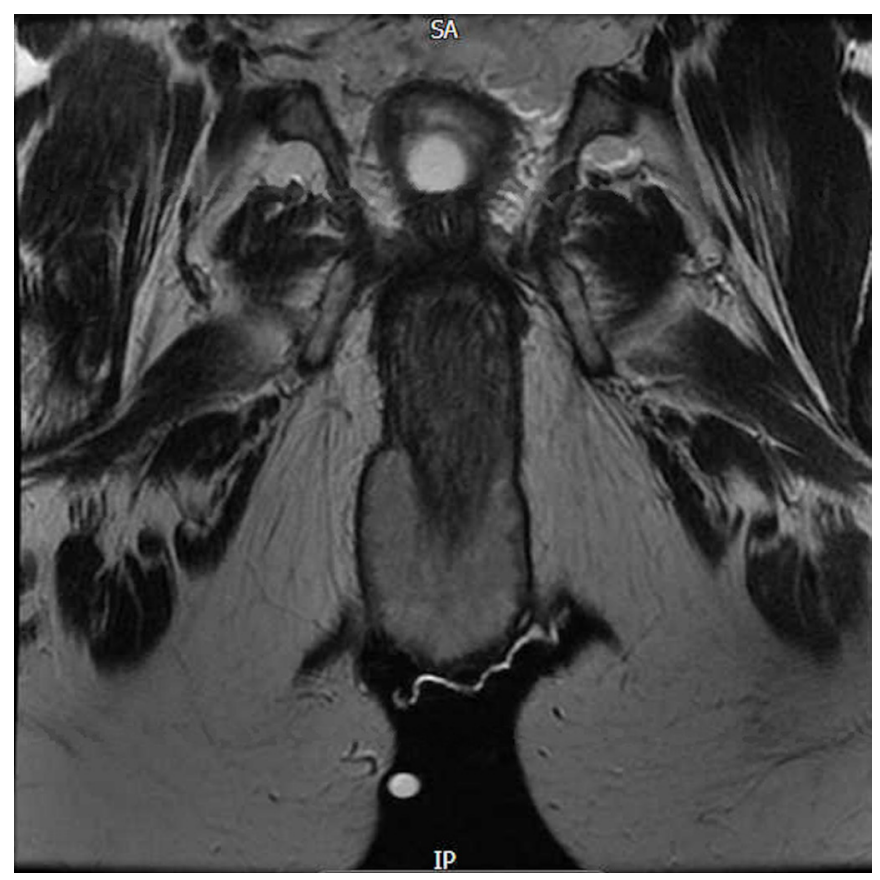

Figure 2 MRI pelvis - coronal view demonstrating a severe uterine prolapse. There was abnormal hyperintense tissue centred on the usual position of the cervix in keeping with tumour. This had a maximum dimension of approximately $9 \mathrm{~cm}$ and filled the vaginal fornices circumferentially. There was no endometrial thickening. It was not possible to assess the parametria due to the degree of prolapse. There was no evidence of lymphadenopathy or metastases. IP, inferior/ superior; SA, superior/anterior.

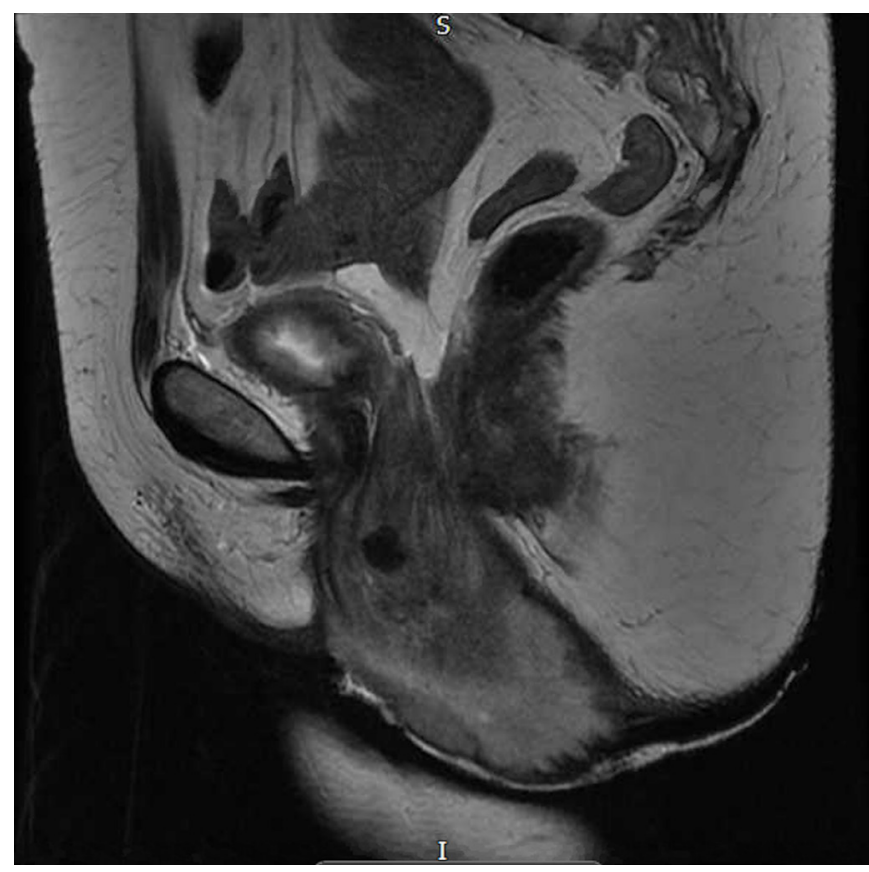

Figure 3 MRI pelvis—saggital view demonstrating a severe uterine prolapse. There was abnormal hyperintense tissue centred on the usual position of the cervix in keeping with tumour. This had a maximum dimension of approximately $9 \mathrm{~cm}$ and filled the vaginal fornices circumferentially. There was no endometrial thickening. It was not possible to assess the parametria due to the degree of prolapse. There was no evidence of lymphadenopathy or metastases.

cytokeratin positive. The histopathological conclusion was that of an endometrial stromal sarcoma (ESS). A soft tissue sarcoma specialist opinion was sought and positron emission tomography (PET) scan requested.

The PET scan reported an extremely fluorodeoxyglucose (FDG) avid prolapsed cervical tumour (maximum standardised uptake value SUVmax $=28.1$ ) with an indeterminate $8 \mathrm{~mm}$ mildly FDG avid (SUVmax=3.0) distal left common iliac lymph node and an ill-defined subcentimetre mildly FDG avid aortocaval lymph node more superiorly and anterior to the L3/4 disc space. There was no evidence of distant metastases. The nature and primary origin of the sarcomatous tumour was deemed uncertain (figures 4 and 5).

Following gynaecological oncology and sarcoma multidisciplinary team (MDT) discussions, the recommendation was made for surgical management. The patient underwent laparoscopic hysterectomy, bilateral salpingo-oophorectomy and omentectomy. Lymph node assessment was unremarkable and therefore a decision was taken not to proceed with systematic lymphadenectomy, in order to reduce surgical-related morbidity and in view of tumour aetiology. Additionally, in our department the sensitivity and specificity of PET scan is high, hence we did not want to subject the patient to dual treatment modality given the fact that she would have needed further adjuvant treatment. This was also the collective view of the MDT, which recommended to assess and excise only the suspicious lymph nodes. On intra-abdominal assessment, the uterus was found to be completely prolapsed, pulling the ureters, round ligaments, fallopian tubes and associated vessels into the vagina (figures 6 and 7). The pelvic sidewalls were opened with skeletonisation of the ureters, internal and external iliac vessels and securing of the uterine arteries at origin with development of the avascular 


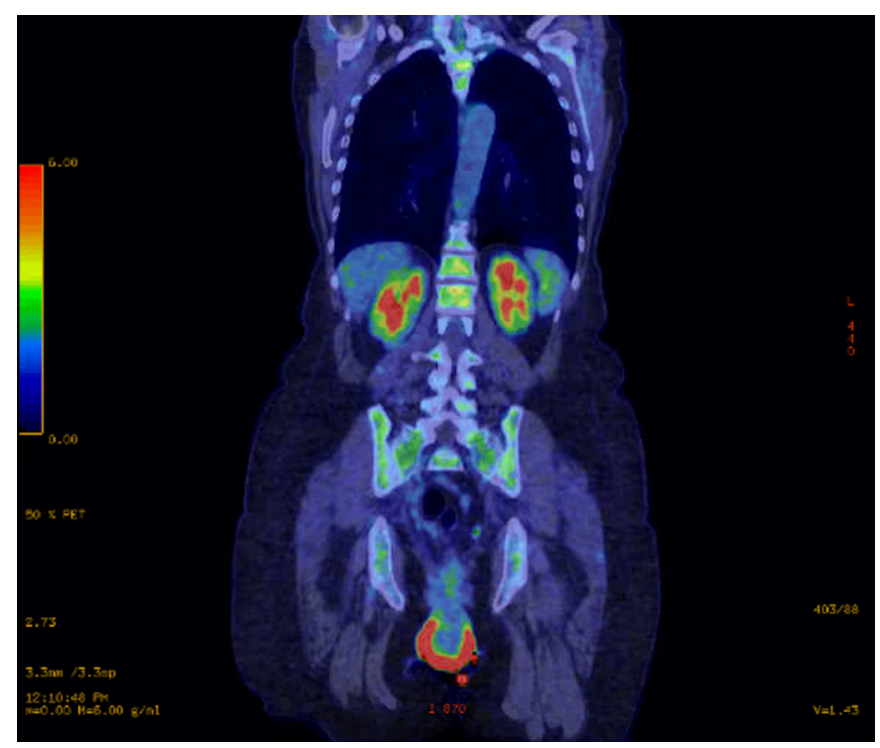

Figure 4 PET CT—coronal view demonstrating an extremely avid prolapsed tumour (SUVmax=28.1). PET, positron emission tomography. SUV, standardized uptake value.

pelvic spaces. Additional ureterolysis and bladder reflection was performed, and a trial made to reduce the prolapse into the abdominal cavity (figure 8). Transverse laparotomy for colpotomy with retrograde modified Hudson and specimen delivery was performed to ensure cancer hygiene and avoid tumour spillage. The specimen was composed of the tumour, uterus, cervix, tubes, ovaries, $1.5-2 \mathrm{~cm}$ vaginal cuff and $2.5-3 \mathrm{~cm}$ parametrium bilaterally (figure 9). The procedure was completed in routine fashion and the patient subsequently made an uneventful recovery with standard antibiotic and dalteparin prophylaxis. She was discharged with an indwelling catheter in situ, which was successfully removed several weeks later.

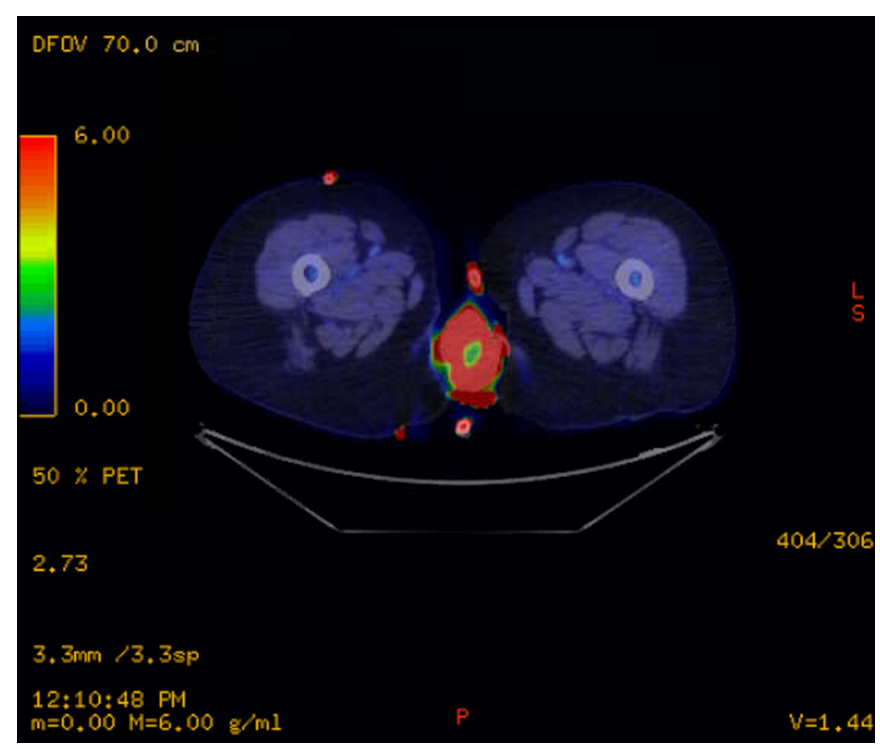

Figure 5 PET CT—axial view demonstrating an extremely avid prolapsed tumour (SUVmax=28.1). PET, positron emission tomography; SUV, standardized uptake value.

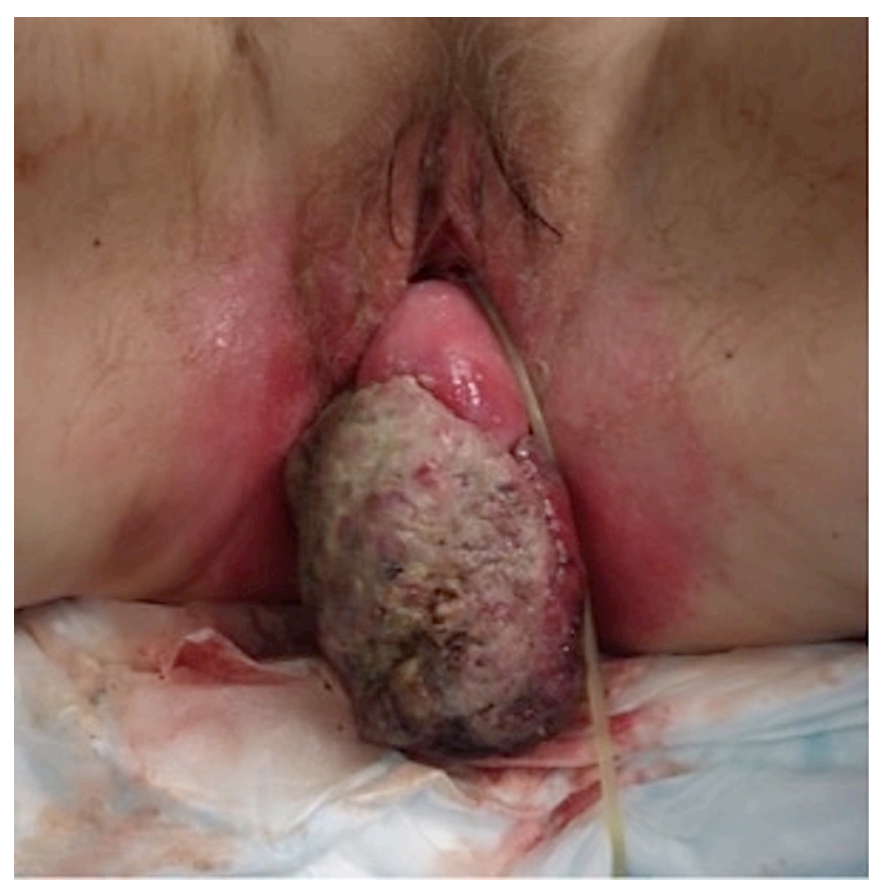

Figure 6 The tumour viewed preoperatively causing procidentia.

\section{OUTCOME AND FOLLOW-UP}

Final histology reported a normal size uterus with a large, nodular, cauliflower-like sarcomatous mass extending out from the external os measuring $7 \mathrm{~cm}$ in maximum diameter (figure 10). On sectioning, it was external to the uterus and indistinguishable from the cervix, enveloping and partly eroding into the cervical and uterine walls as well as the vaginal cuff. The tumour was present at the vaginal margins of this excision and within the parametrium. The endocervical canal was not identified and the endometrium was atrophic. The distribution suggested that this tumour was extending from the outside inwards and secondarily involving the vagina, cervix and lower uterus. There was no unequivocal evidence that the tumour represented an origin in

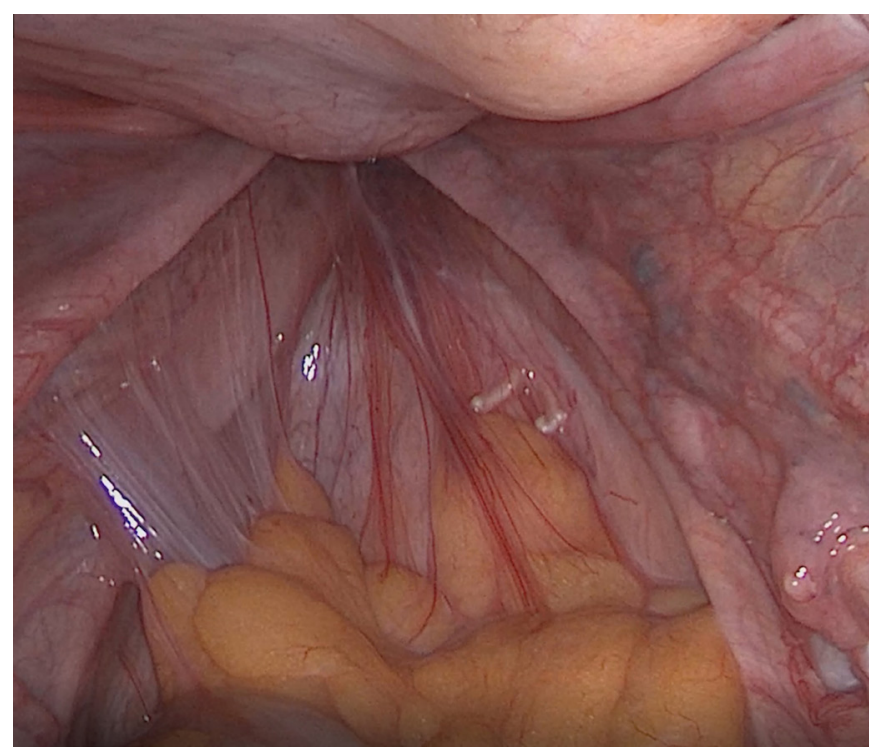

Figure 7 Laparoscopic intraabdominal view of the pelvis demonstrating both fallopian tubes and round ligaments descending into the region of the vagina with absence of the prolapsed uterus. 


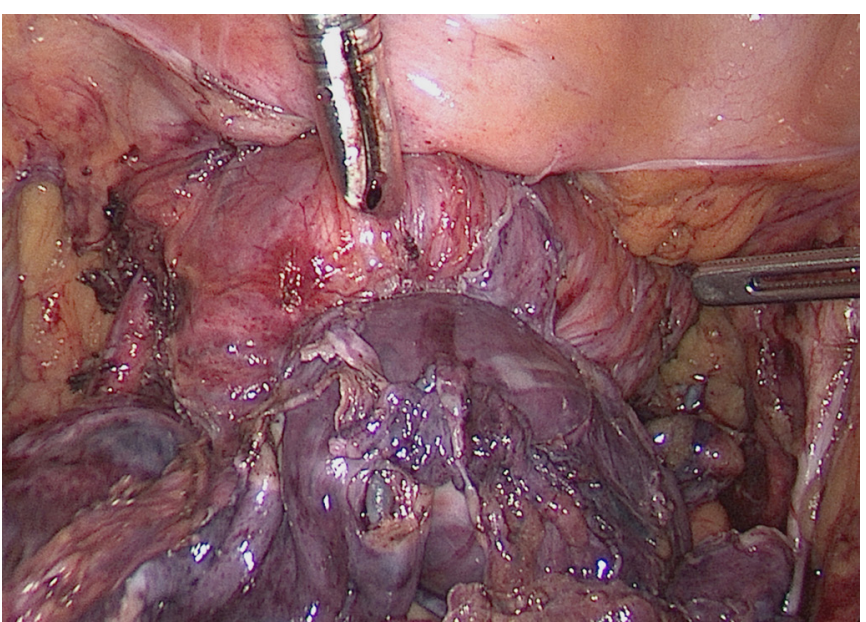

Figure 8 Laparoscopic intrabdominal view of the pelvis on having replaced the uterus into the cavity and following devascularisation. Further tumour within the vagina was seen to distort the bladder and posed surgical challenges regarding safe colpotomy.

the gynaecological tract. As a result, no pathological staging was performed (figures 11 and 12).

In view of high risk of recurrence, following review at the gynaecological oncology and sarcoma MDTs, the patient was considered for further examination under anaesthesia with vaginal biopsy or upper vaginectomy. The patient was keen to avoid further surgery and was therefore directly referred on to clinical oncology for consideration of adjuvant radiotherapy. Repeat PET and MRI scans showed no absolute evidence of recurrent disease.

Adjuvant pelvic radiotherapy 50 Gy in 25 fractions followed by vaginal vault brachytherapy 8 Gy in 2 fractions, were given to reduce her risk of recurrence and were completed 4 months after the surgery. The patient reports being well, remaining asymptomatic and had a PET scan 4 months after completion of the adjuvant treatment which showed no residual FDG avid disease.

\section{DISCUSSION}

Cancers of unknown origin consist of metastatic malignancies without a defined primary site. Heterogeneity is the most common characteristic of these tumours, as their presentation follows no specific pathway regarding type of the malignancy, spread of disease and response to treatment. Such tumours can be classified into those comprising of epithelial cells and those

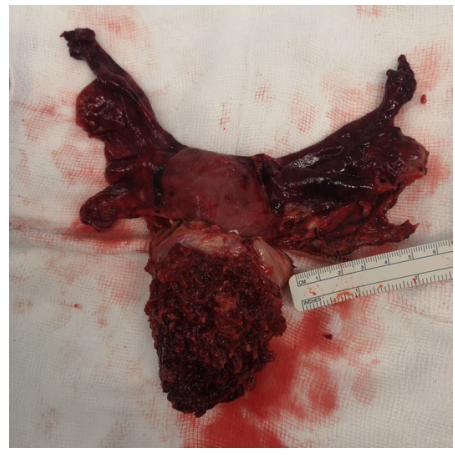

Figure 9 Specimen following type three radical hysterectomy including the tumour, uterus, cervix, tubes, ovaries, vaginal cuff and the parametrium bilaterally.

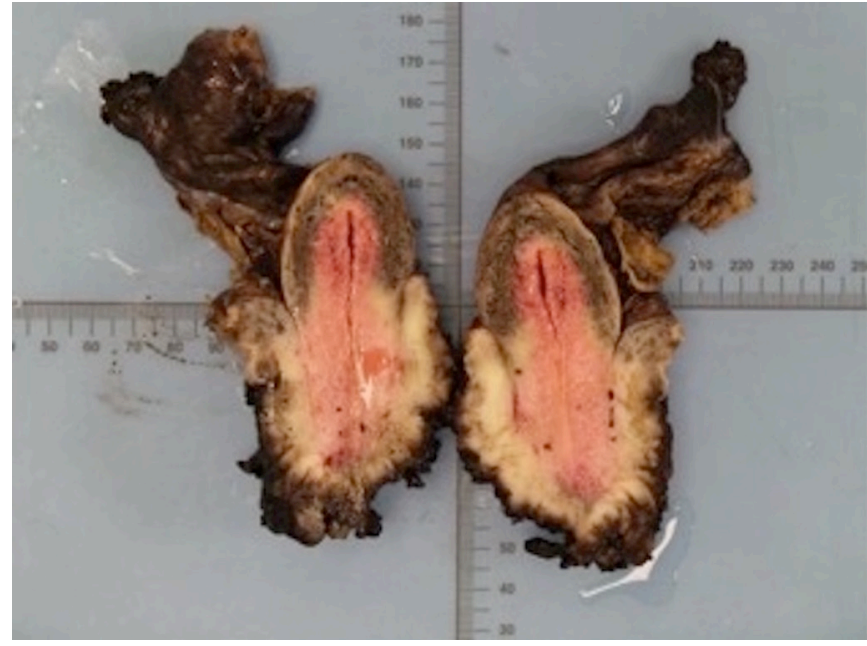

Figure 10 Macroscopic appearance of the histopathological specimen. The tumour was seen to extensively envelope the lower uterus, the cervix and the vaginal cuff.

deriving from non-epithelial cells such as melanomas, sarcomas, lymphomas and germ-cell tumours. ${ }^{1}$

Sarcomas are rare mesenchymal tumours that can present in any part of the human body. They can affect people at any age, but they are more common in middle-aged and older individuals. Patients with high-grade tumours have an increased risk of developing metastatic disease, which will require systemic treatment.

Approximately $75 \%$ of sarcomas arise from the soft tissue and characterised by a low incidence in all populations. Sarcomas in the female genital tract account for approximately 3\%-4\% of all gynaecological malignancies with uterine soft tissue sarcoma representing approximately $83 \%$ of all gynaecological sarcomas. ${ }^{78}$ The most common histological subtype is leiomyosarcoma (63\%) followed by ESS (21\%) and high-grade or undifferentiated uterine sarcoma (UUS) $(16 \%) .{ }^{9}$ Cervical sarcomas account for less than $1 \%$ of all cervical malignancies. ${ }^{10}$

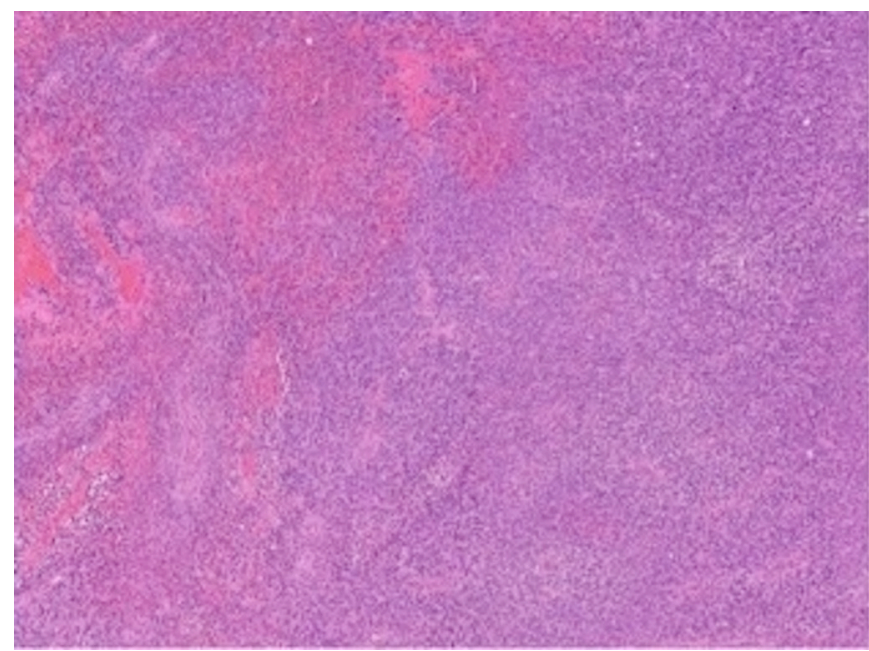

Figure 11 Microscopic histopathological appearance showing largely diffuse architecture. The tumour was seen to contain mainly ovoid and spindle shaped cells with prominent nuclear pleomorphism and high mitotic rate, with extensive necrosis and no obvious epithelial differentiation. It was noted to be present in the parametrium and at the resection margins of the specimen. 


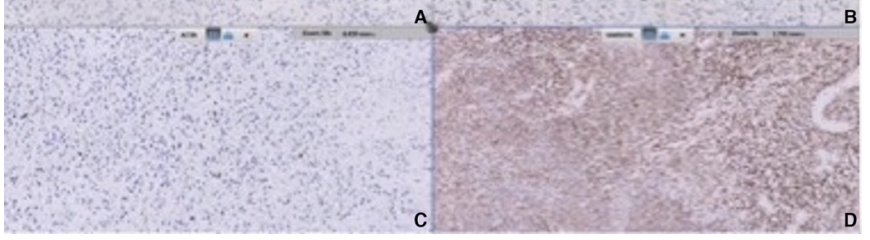

Figure 12 Immunohistochemistry histopathological examinations showed immunonegativity for MNF116 (A), weak staining for musclespecific desmin (B) and actin (C) and patchy positive staining for vimentin (D).

UUS and undifferentiated cervical sarcoma are even rarer tumours which lack specific line of differentiation and the diagnosis is set after exclusion of more common types of highgrade tumours. In our case, even after multiple examinations of specialist sarcoma pathologists and gynaecological pathologists followed by consensus meetings the origin of the tumour remained undefined. ${ }^{1011}$

The development of soft tissue sarcomas has not directly been linked to any specific aetiology. Previous exposures to ionising radiation, agent orange or to certain other chemicals such as vinyl chloride, chlorophenols, phenoxyacetic acids and arsenic have been implicated. However, it has been proven that there is an increased incidence of sarcomas in individuals with a family history of Li-Fraumeni syndrome, who are carrying inherited TP53 tumour suppressor gene mutations or in those with familial retinoblastoma due to RB gene mutations. Other inherited diseases such as neurofibromatosis and Gardner's syndrome are also associated with an increased lifetime risk of soft tissue sarcomas due to the prevalence of several gene mutations. ${ }^{12}$

The signs and symptoms that can guide a patient to ask for medical advice can vary depending on the site and pattern of spread of the disease. Most commonly, regarding disease located in the female genital tract, the patient might report pelvic or abdominal pain, bleeding or abnormal discharge from the vagina or simply undetermined discomfort. ${ }^{2}$ Secondary to a growing mass, the patients may experience changes in their bladder or bowel habit and finally, in advanced stages, more generalised signs like fatigue, loss of appetite or systematic symptoms may be present. ${ }^{513}$

The diagnosis is based on a combined, thorough clinical assessment of the patient, imaging and extensive histopathological investigations. ${ }^{14}{ }^{15}$ Unfortunately, there are no clinically validated tumour markers to suspect and detect such neoplasms. The heterogeneity and undetermined nature of those malignancies necessitate multiple immunohistochemical examinations, which may provide precise information for the histological classification and the origin of the tumour. Chromosomal analysis of the tissue may also facilitate the detection of the primary tumour on a molecular basis. ${ }^{16}$

The cornerstone in the treatment of sarcoma is primary surgical resection, achieving macroscopically and, if possible, microscopically negative margins. Adjuvant or neoadjuvant treatment can also be offered to improve the outcome and prognosis. ${ }^{1517}$ Adjuvant radiotherapy has been correlated with improved survival in UUS patients. ${ }^{18}$ Chemotherapy is the preferred modality in locally advanced or metastatic disease where surgical excision is not feasible. The responsiveness of the disease to further treatment is intimately related to the histological subtype. ${ }^{19}$ However, primary tumours may remain undetected in such cases where histology based management is not an option. Here, further treatment is offered empirically, considering the performance status of the patient. ${ }^{20}$

The most determinant prognostic factors for sarcoma cases are the histological subtype and the grade of differentiation of the tumour; with high-grade tumours being associated with poorer outcomes. For tumours of unknown primary origin, the stage of the disease at the time of diagnosis and the aggressiveness of the tumour, play the most important prognostic role. ${ }^{15}$

To our knowledge, there are no reported cases of poorly differentiated sarcoma of unknown primary origin associated with stage IV pelvic organ prolapse in the literature. Leem et al had presented a case of a basaloid squamous cell carcinoma of the uterine cervix coexisting with undifferentiated sarcoma causing uterine prolapse, which was removed vaginally. ${ }^{21}$

In our case, although most surgeons would have performed a laparotomy, we proceeded with laparoscopic surgery as there was no confirmation of a cervical malignancy and the initial biopsy had indicated an ESS, in consideration of the Laparoscopic Approach to Cervical Cancer (LACC) trial. ${ }^{22}$ Our patient had already faced the grave consequences of the atypical and late presentation of a rare tumour, which, combined with the COVID-19 pandemic situation and her COVID-19 infection, caused a significant delay in her cancer pathway management. The continuous odoriferous discharge, the size of the prolapsed tumour and the secondary urinary retention had already caused a severe deterioration in the patient's quality of life. By laparoscopy, we aimed to acquire clear excision margins, to minimise the possibility of any further intraoperative or postoperative complications and to reduce the length of hospitalisation and subsequent recovery time. In consideration of the potential requirement for future adjuvant treatment, by minimising the potential complications and postoperative recovery time, we could prevent any further delay in her holistic management.

On accessing the peritoneal cavity, this revealed a challenging operating field. All the surrounding supportive tissues of the uterus and cervix as well as both adnexa had been pulled caudally through the vagina. Although this might be a common intraabdominal appearance associated with stage 4 uterine prolapse, in this case, the size of the tumour had caused the round and ovarian ligaments to completely disappear into the vagina, with the structures relatively fixed secondary to mass effect. After dissecting the round ligament, the development of the avascular spaces in the retroperitoneal spaces was extremely challenging as the ureters and the iliac vessels were pulled medially and caudally along with the surrounding tissues, causing complete disruption of the anatomical margins.

The laparoscopic approach offered us an advantageous and clearer vision in the surgical field and was extremely helpful in that step of the procedure. Type 3 radical hysterectomy was performed following devascularisation of the uterus by division of the uterine arteries at origin. By achieving the hypoperfusion of the tumour we were able to minimise the intraoperative blood loss. After a gentle unsuccessful attempt to replace the uterus into pelvis due to its size, we proceeded to transverse laparotomy to perform anterior colpotomy and safely retrieve the specimen amidst surgical challenges; preventing tumour spillage while the pneumoperitoneum was still in situ and with respect to oncological hygiene. ${ }^{15}$

The effective management of this case was a result of continuous MDT consensus, precise surgical management and close surveillance of the patient. Complicated, rare cases like this, 
which cannot be classified into a specific category of origin, present a challenging approach to management where guidelines are not available. Response and outcomes are difficult to define and hence such cases deserve innovative perspective and treatment while thinking 'outside the box'.

This case became a true inspiration for our team to envision the concept of a 'Rare Incidence Bank', where all rare cases would be fully recorded by each institution including the presentation, diagnostic pathway, treatment method and outcome, complications, recurrences and survival trends. The multicentred collected data could be used to organise evidence-based programmes, which will assess the outcomes and the efficacy of a variety of practices. Integrated knowledge deriving from evidence-based programmes will offer optimal healthcare to the patients involved while enhancing the healthcare professional's proficiency in the management of rare cases.

\section{Learning points}

- Poorly differentiated sarcomas of unknown primary origin constitute rare malignancies that can appear with unusual and challenging presentations, which have been further potentiated during the COVID-19 pandemic in later disease stages.

- Their undetermined content and wide heterogeneity poses severe limitations in their diagnosis and management with an increased lifetime risk of recurrence and poor prognosis.

- Histopathology and immunohistochemistry investigations play a fundamental role in the diagnosis.

- Effective management is a combination of continuous multidisciplinary team consensus, precise individualised surgical management and close surveillance of the patient.

- Systemic record keeping of these cases could contribute to acquisition of clear management guidelines and offer optimal therapy to patients in the future.

Contributors HSM conceived the manuscript, HSM and SS performed senior review, CP and SS composed the manuscript.

Funding The authors have not declared a specific grant for this research from any funding agency in the public, commercial or not-for-profit sectors.

Competing interests None declared.

Patient consent for publication Consent obtained directly from patient(s).

Provenance and peer review Not commissioned; externally peer reviewed.

Case reports provide a valuable learning resource for the scientific community and can indicate areas of interest for future research. They should not be used in isolation to guide treatment choices or public health policy.

ORCID iDs

Christina Pappa http://orcid.org/0000-0003-2801-2786
Sarah Louise Smyth http://orcid.org/0000-0001-7407-8247

Hooman Soleymani majd http://orcid.org/0000-0003-3293-5321

\section{REFERENCES}

1 Pavlidis N, Fizazi K. Carcinoma of unknown primary (CUP). Crit Rev Oncol Hematol 2009:69:271-8.

2 Tanner EJ, Garg K, Leitao MM, et al. High grade undifferentiated uterine sarcoma: surgery, treatment, and survival outcomes. Gynecol Oncol 2012;127:27-31.

3 Guarnerio J, Riccardi L, Taulli R, et al. A genetic platform to model sarcomagenesis from primary adult mesenchymal stem cells. Cancer Discov 2015;5:396-409.

4 Beisenayeva A, Cialkowska-Rysz A, Zhumaliyeva V. The importance of immunohistochemical studies in the diagnosis of cancer of unknown primary origin. Georgian Med News 2015;249:73-80.

5 Losa F, Soler G, Casado A, et al. SEOM clinical guideline on unknown primary cancer (2017). Clin Trans/ Oncol 2018;20:89-96.

6 Plot L, Dovrish Z, Hadari R, et al. [Cancer of unknown primary site origin--advances in diagnosis and therapy]. Harefuah 2008;147:294-8.

7 Francis M, Dennis NL, Hirschowitz L, et al. Incidence and survival of gynecologic sarcomas in England. Int J Gynecol Cancer 2015;25:850-7.

8 Cree IA, White VA, Indave BI, et al. Revising the WHO classification: female genital tract tumours. Histopathology 2020;76:151-6.

9 Amant F, Coosemans A, Debiec-Rychter M, et al. Clinical management of uterine sarcomas. Lancet Oncol 2009:10:1188-98.

10 Khosla D, Gupta R, Srinivasan R, et al. Sarcomas of uterine cervix: clinicopathological features, treatment, and outcome. Int J Gynecol Cancer 2012;22:1026-30.

11 Philip C-A, Pautier P, Duffaud F, et al. High-grade undifferentiated sarcomas of the uterus: diagnosis, outcomes, and new treatment approaches. Curr Oncol Rep 2014;16:405.

12 Thomas DM, Savage SA, Bond GL. Hereditary and environmental epidemiology of sarcomas. Clin Sarcoma Res 2012;2:13.

13 Pavlidis N. Forty years experience of treating cancer of unknown primary. Acta Oncol 2007:46:592-601.

14 Moller AKH, Loft A, Berthelsen AK, et al. 18F-FDG PET/CT as a diagnostic tool in patients with extracervical carcinoma of unknown primary site: a literature review. Oncologist 2011;16:445-51.

15 National Institute for Health and Clinical Excellence. Improving outcomes for people with sarcoma, 2006. Available: www.nice.org.uk/guidance/csgsarcoma/evidence/ improving-outcomesfor-people-with-sarcoma-the-manual [Accessed Dec 2016].

16 Lin F, Liu H. Immunohistochemistry in undifferentiated neoplasm/tumor of uncertain origin. Arch Pathol Lab Med 2014;138:1583-610.

17 Woll PJ, Reichardt P, Le Cesne A, et al. Adjuvant chemotherapy with doxorubicin, ifosfamide, and lenograstim for resected soft-tissue sarcoma (EORTC 62931): a multicentre randomised controlled trial. Lancet Oncol 2012;13:1045054.

18 Cabrera S, Bebia V, Acosta U, et al. Survival outcomes and prognostic factors of endometrial stromal sarcoma and undifferentiated uterine sarcoma. Clin Trans/ Oncol 2021;23:1210-9.

19 Casali PG, Blay JY. ESMO/CONTICANET/EUROBONET consensus panel of experts. soft tissue sarcomas: ESMO clinical practice guidelines for diagnosis, treatment and follow-up. Ann Oncol 2010;21:v198-203.

20 Gamboa AC, Gronchi A, Cardona K. Soft-tissue sarcoma in adults: an update on the current state of histiotype-specific management in an era of personalized medicine. CA Cancer J Clin 2020;70:200-29.

21 Lemm M, Słowik Łukasz, Cichoń B, et al. Basaloid squamous cell carcinoma of the uterine cervix coexisting with undifferentiated sarcoma. Prz Menopauzalny 2020;19:192-4.

22 Ramireza PT, Frumovitza M, Parejab R. Phase III randomized trial of laparoscopic or robotic versus abdominal radical hysterectomy in patients with early-stage cervical cancer: LACC Trial. Abstract presented at the 49th Annual Meeting of the Society of Gynecologic Oncology; March 24-27, New Orleans, LA, USA, 2018. 
Copyright 2022 BMJ Publishing Group. All rights reserved. For permission to reuse any of this content visit https://www.bmj.com/company/products-services/rights-and-licensing/permissions/

BMJ Case Report Fellows may re-use this article for personal use and teaching without any further permission.

Become a Fellow of BMJ Case Reports today and you can:

- Submit as many cases as you like

Enjoy fast sympathetic peer review and rapid publication of accepted articles

Access all the published articles

Re-use any of the published material for personal use and teaching without further permission

Customer Service

If you have any further queries about your subscription, please contact our customer services team on +44 (0) 2071111105 or via email at support@bmj.com.

Visit casereports.bmj.com for more articles like this and to become a Fellow 